\title{
ESTUDIO COMPARATIVO DEL EFECTO ANTIOXIDANTE DE Ipomoea batata L. “CAMOTE MORADO” EN POLVO LIOFILIZADO Y ATOMIZADO
}

\author{
Juan Tovar ${ }^{\text {a }}$, Diana Vargas ${ }^{\mathrm{a}}$, Ricardo Alaya ${ }^{\mathrm{a}}$, Jhonnel Samaniego*a, \\ Jenny Huerta ${ }^{a}$, Miguel Inocente ${ }^{a}$
}

\begin{abstract}
RESUMEN
El objetivo del estudio fue determinar la actividad antioxidante en el polvo liofilizado y atomizado de Ipomoea batata L. por los métodos DPPH y FRAP, en la variedad proveniente de la zona de Piura conocida como "camote morado". Se utilizaron dos métodos de secado, por atomización y liofilización. Para determinar la capacidad antioxidante se utilizaron los métodos DPPH y FRAP. Se realizó la prueba de solubilidad con agua, etanol y metanol. Se obtuvo como resultados los valores de 23,23 $\mu$ mol Trolox equivalentes/g para DPPH y 20,18 $\mu$ mol Trolox equivalentes/g para FRAP en la muestra liofilizada y valores de 5,24 $\mu \mathrm{mol}$ Trolox equivalentes/g para DPPH y 4,96 $\mu$ mol Trolox equivalentes/g para FRAP en la muestra atomizada. En conclusión, los resultados muestran que la capacidad antioxidante de la variedad de Ipomoea Batatas L. (camote morado) presenta una gran diferencia en los resultados según el método de secado utilizado, demostrando una mayor capacidad antioxidante el secado por liofilizado.
\end{abstract}

Palabras clave: Ipomoea batata L., camote morado, actividad antioxidante, DPPH, FRAP

\section{COMPARATIVE STUDY OF THE ANTIOXIDANT EFFECT OF Ipomoea batata L. "PURPLE SWEET POTATO" IN LYOPHILIZED AND ATOMIZED POWDER}

\begin{abstract}
The objective of the study was to determine the antioxidant activity in the lyophilized and atomized powder of Ipomoea batata L. by the DPPH and FRAP methods, in the variety from the Piura area known as "purple sweet potato". Two drying methods were used, spray and lyophilization. To determine the antioxidant capacity, the DPPH and FRAP methods were used. The solubility test was carried out with water, ethanol and methanol. The results were the values of $23.23 \mu \mathrm{mol}$ Trolox equivalents / $\mathrm{g}$ for DPPH and $20.18 \mu \mathrm{mol}$ Trolox equivalents
\end{abstract}

\footnotetext{
a Facultad de Ciencias de la Salud, Universidad María Auxiliadora, Av. Canto Bello 431, San Juan de Lurigancho, Lima 15408, Perú. jhonnel.samaniego@uma.edu.pe
} 
/ $\mathrm{g}$ for FRAP in the lyophilized sample and values of $5.24 \mu \mathrm{mol}$ Trolox equivalents / $\mathrm{g}$ for DPPH and $4.96 \mu \mathrm{mol}$ Trolox equivalents / $\mathrm{g}$ for FRAP in the atomized sample. In conclusion, the results show that the antioxidant capacity of the variety of Ipomoea Batatas L. (Purple Sweet Potato) presents a great difference in the results according to the drying method used, with freeze-dried drying demonstrating a higher antioxidant capacity.

Key words: Ipomoea batata L., purple sweet potato, antioxidant activity, DPPH, FRAP

\section{INTRODUCCIÓN}

Los radicales libres se pueden liberar durante el metabolismo humano, y además se producen por diversos contaminantes ambientales, como por ejemplo los atmosféricos, acuáticos, de suelos, radiaciones como las ultravioleta y gamma, entre otros. Podemos incluir el metabolismo de algunos químicos y el elevado estrés físico o psíquico que pueda tener un individuo ${ }^{1}$. Según lo estipulado por Núñez (2011), se han podido estudiar alrededor de 100 enfermedades y su relación con el desbalance del sistema oxidativo, entre otras tenemos: enfermedades cardiovasculares, gástricas, respiratorias, neurológicas, del sistema endocrino y cáncer, las cuales se pueden prevenir o ser reducidas con los compuestos antioxidantes presentes en algunos alimentos ${ }^{2}$. En el mundo existen diversos estudios que evalúan la capacidad antioxidante del camote en distintas variedades, debido a que presenta componentes fenólicos que le puedan brindan estas propiedades ${ }^{3,4,5}$. El camote es un alimento que no tiene un consumo habitual, uno de los motivos podría ser el desconocimiento de los beneficios de este importante alimento que pueda tener para la salud y la falta de difusión de la amplia variedad de camotes con la que cuenta el Perú, existiendo entre 250 a 300 variedades ${ }^{6}$. Se determinó el efecto antioxidante de este camote por dos métodos: Poder antioxidante del ion férrico (FRAP) y 2,2-Difenil -1-picrilhidrazilo (DPPH), teniendo en cuenta que los tratamientos de secado afectan la presencia de los componentes y de la capacidad antioxidante presente en la muestra, lo cual ha sido reportado en varios estudios ${ }^{7,8,9}$.

El objetivo de la presente investigación es determinar la actividad antioxidante en el polvo liofilizado y atomizado de Ipomoea batata L. (camote morado) por los métodos DPPH y FRAP.

\section{PARTE EXPERIMENTAL}

Recolección y certificación taxonómica de la muestra para el estudio: La recolección del Ipomoea batata L., camote morado, se realizó en la Región Piura, Provincia de Piura, Distrito de Catacaos, Centro Poblado la Unión, Anexo Chalaco. Esta se encuentra a una altitud de 60 m.s.n.m. Se recolectó un promedio de $20 \mathrm{~kg}$ de muestra, a la cual se le realizó la clasificación botánica de la especie por un consultor botánico inscrito en el registro de profesionales que realizan la identificación taxonómica de especímenes y productos de la flora $\mathrm{RD} \mathrm{N}^{\circ} 0311$ 2013-MINAGRI. 
Secado por liofilizado: Las raíces de la Ipomoea batata L., camote morado, fueron lavadas con abundante agua, para eliminar impurezas y tener una muestra homogénea. Luego de ello se procedió a pesar las raíces, para después cortarlas en láminas delgadas y colocarlas en las bandejas del equipo liofizador. El proceso de liofilizado se realizó por 12 horas a una temperatura de $-18{ }^{\circ} \mathrm{C}$ en un equipo STELLAR ${ }^{\circledR}$ Laboratory Freeze Dryer | Millrock Technology, Inc. Las láminas del liofilizado de Ipomoea batata L., camote morado, fueron trituradas hasta pulverizarlo con ayuda de un mortero y luego almacenado en bolsa de aluminio a temperatura controlada entre 15 a $20^{\circ} \mathrm{C}$. Siendo su humedad relativa entre 7 a $15 \%$.

Secado por atomizado: Las raíces de Ipomoea batata L., camote morado, se lavaron con abundante agua, para eliminar y tener una muestra homogénea. Luego se procedió a triturar y tamizar por malla $\mathrm{N}^{\circ} 20$. Después se llevó a atomizar teniendo en cuenta la siguiente proporción $90 \%$ de pulpa de raíz Ipomoea batata L., camote morado y $10 \%$ de maltodextrina (Maltogill 20, Dextrose Equivalent-DE 20). Se realizó el secado a través del equipo A/S NIRO NF ATOMIZER ${ }^{\circledR}$ spray-drying, hecho en Dinamarca con los siguientes parámetros de proceso. Temperatura de ingreso: 160 a $180{ }^{\circ} \mathrm{C}$, temperatura de salida: 85 a $95{ }^{\circ} \mathrm{C}$ a una velocidad de un litro por hora. El polvo atomizado fue almacenado en bolsa de alupol de 5 gramos, a temperatura controlada entre 15 a $20^{\circ} \mathrm{C}$, bajo humedad relativa $<$ a $17 \%$ para su posterior procesamiento y análisis.

Prueba de solubilidad: En tres tubos de ensayo se colocó una cantidad de muestra (10 mg), liofilizado y atomizado de la raíz de Ipomoea batata L. (camote morado) y se agregó a cada uno de los tubos de ensayo $1 \mathrm{~mL}$ del solvente de diferente polaridad. Luego se agitó con la ayuda de una bagueta y se observó los resultados. Los solventes utilizados fueron agua $\left(\mathrm{H}_{2} \mathrm{O}\right)$, etanol $(\mathrm{EtOH})$ y metanol $(\mathrm{MeOH})$.

Actividad de captación de radicales DPPH: La actividad antioxidante se determinó mediante el efecto de eliminación de radicales libres DPPH utilizando el reactivo de Trolox como control positivo, según el método (Nwaehujor et al., 2014). La curva de calibrado de Trolox se preparó en un rango de concentraciones $(50-1000 \mu \mathrm{mol})$. Se añadieron 290 $\mu \mathrm{l}$ de solución de DPPH metanólico $\left(1 \times 10^{5} \mathrm{M}\right)$ y la mezcla se incubó durante una hora a temperatura ambiente a leve agitación y protegido de la luz. La absorbancia se leyó a $517 \mathrm{~nm}$ y los datos se adquirieron y procesaron utilizando el software Gen5TM de BioTek (BioTek Instruments Inc.). La actividad de captación de radicales libres se determinó de acuerdo con la ecuación de \% de Actividad Antioxidante $(\mathrm{AA})=100-[\{($ Absorbancia de la muestraAbsorbancia del blanco $) \times 100\} /$ Absorbancia control] publicada anteriormente ${ }^{12}$.

Ensayo de potencia férrica antioxidante reductor FRAP: Se determinó el potencial antioxidante total de una muestra utilizando el método de poder antioxidante reductor férrico (FRAP) de Benzie y Strain (1996). Este método se basa en reducir el poder de un compuesto antioxidante. Un potencial antioxidante reducirá al ion férrico $\left(\mathrm{Fe}^{3+}\right)$ al ion ferroso $\left(\mathrm{Fe}^{2+}\right)$ a bajo $\mathrm{pH}$; las últimas formas un complejo azul ( $\left.\mathrm{Fe}^{2+} / \mathrm{TPTZ}\right)$, medido a $593 \mathrm{~nm}$. El reactivo FRAP se preparó recientemente, mezclando tampón de acetato $0,3 \mathrm{M}(\mathrm{pH} \mathrm{3,6),} \mathrm{TPTZ} 10$

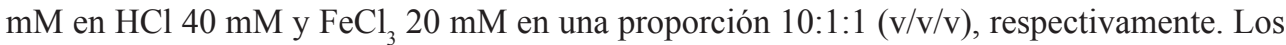


estándares de Trolox se prepararon en un rango de $50 \mu \mathrm{M}$ a $1000 \mu \mathrm{M}$ en una mezcla de $\mathrm{H} 2 \mathrm{O} /$ etanol (75:25). El ensayo se llevó a cabo mediante la adición de $10 \mu l$ de muestra/ estándares y blanco en una microplaca de 96 pocillos y luego se agregó $290 \mu \mathrm{l}$ de reactivo FRAP. Después de 15 min de incubación a $37^{\circ} \mathrm{C}$ y agitación, se leyó la absorbancia a $593 \mathrm{~nm}$. Todas las muestras se realizaron por triplicado. Los resultados fueron comparados con una curva estándar preparada diariamente con diferentes concentraciones de Trolox y se expresaron los resultados como $\mu \mathrm{mol}$ de equivalentes de Trolox $(\mathrm{TE})^{12}$.

\section{RESULTADOS Y DISCUSIÓN}

En la prueba de solubilidad se determinó si es miscible al producirse una mezcla homogénea entre el polvo liofilizado y atomizado de la raíz de Ipomoea batata L., camote morado y el solvente asignado, encontrándose los siguientes resultados según tabla 1.

Tabla 1. Prueba de solubilidad del liofilizado y atomizado de la raíz de Ipomoea batata L., camote morado.

\begin{tabular}{lc}
\hline \multicolumn{1}{c}{ Reactivos } & Resultados \\
\hline \multicolumn{1}{c}{ 10mg de muestra en $1 \mathrm{~mL}$. de } & solvente \\
\hline Liofilizado en agua $\left(\mathrm{H}_{2} \mathrm{O}\right)$ & Miscible \\
Liofilizado en etanol $(\mathrm{EtOH})$ & Miscible \\
Liofilizado en metanol $(\mathrm{MeOH})$ & Miscible \\
Atomizado en agua $\left(\mathrm{H}_{2} \mathrm{O}\right)$ & Miscible \\
Atomizado en etanol $(\mathrm{EtOH})$ & Miscible \\
Atomizado en metanol $(\mathrm{MeOH})$ & No miscible \\
\hline
\end{tabular}

En la tabla 2 se muestran los resultados del ensayo DPPH, se evalúa la actividad antioxidante para neutralizar un radical y son expresados en $\mu \mathrm{M}$ Trolox equivalente/g. Cada muestra fue analizada por duplicado en grupos de camote morado atomizado y liofilizado.

Tabla 1. Determinación de la actividad antioxidante DPPH.

\begin{tabular}{|c|c|c|c|c|c|c|c|c|}
\hline \multirow[t]{2}{*}{ Grupo } & \multirow[t]{2}{*}{$\mathrm{N}$} & \multirow{2}{*}{$\begin{array}{c}\text { Media } \\
\mu \mathrm{M} \\
\text { Trolox/g }\end{array}$} & \multirow{2}{*}{$\begin{array}{l}\text { Desv. } \\
\text { Estándar }\end{array}$} & \multirow{2}{*}{$\begin{array}{l}\text { Desv. } \\
\text { Error }\end{array}$} & \multicolumn{2}{|c|}{$95 \%$ IC } & \multirow[t]{2}{*}{ Mínimo } & \multirow[t]{2}{*}{ Máximo } \\
\hline & & & & & $\begin{array}{l}\text { Límite } \\
\text { inferior }\end{array}$ & $\begin{array}{l}\text { Límite } \\
\text { superior }\end{array}$ & & \\
\hline Patrón & 4 & 0,0000 & 0,0000 & 0,0000 & 0,0000 & 0,0000 & 0,0000 & 0,0000 \\
\hline Atomizado & 4 & 5,2407 & 2,1591 & 1,0796 & 1,8051 & 25,0996 & 2,5474 & 7,4394 \\
\hline Liofilizado & 4 & 23,2251 & 1,1780 & 0,5890 & 21,3506 & 22,3816 & 22,2178 & 24,5318 \\
\hline Total & 12 & & & & & & & \\
\hline
\end{tabular}


En la tabla 3 se muestra los resultados del ensayo FRAP un complejo de color amarillo que es el Fe ${ }^{3+}$-TPTZ (2,4,6-tripiridil-s-triazina), que es reducido al complejo azul de Fe2+-TPTZ por un electrón donador. Se realizó la curva estándar con el patrón Trolox. Cada muestra fue medida por duplicado en grupos de camote atomizado y camote liofilizado. Los resultados descriptivos se reportan en unidades $\mu \mathrm{M}$ Trolox/g.

Tabla 3. Datos descriptivos de la capacidad antioxidante FRAP ( $\mu \mathrm{M}$ Trolox/g).

\begin{tabular}{|c|c|c|c|c|c|c|c|c|}
\hline \multirow[t]{2}{*}{ Grupo } & \multirow[t]{2}{*}{$\mathrm{N}$} & \multirow[t]{2}{*}{ Media } & \multirow{2}{*}{$\begin{array}{l}\text { Desv. } \\
\text { Estándar }\end{array}$} & \multirow{2}{*}{$\begin{array}{l}\text { Desv. } \\
\text { Error }\end{array}$} & \multicolumn{2}{|c|}{$95 \%$ IC } & \multirow[t]{2}{*}{ Mínimo } & \multirow[t]{2}{*}{ Máximc } \\
\hline & & & & & $\begin{array}{l}\text { Límite } \\
\text { inferior }\end{array}$ & $\begin{array}{c}\text { Límite } \\
\text { superior }\end{array}$ & & \\
\hline Patrón & 4 & 0,0000 & 0,0000 & 0,0000 & 0,0000 & 0,0000 & 0,0000 & 0,0000 \\
\hline Atomizado & 4 & 4,9575 & 0,2351 & 0,1175 & 4,5834 & 5,3316 & 4,6365 & 21,6753 \\
\hline Liofilizado & 4 & 20,1814 & 1,3162 & 0,6581 & 18,0869 & 22,2758 & 18,9487 & 21,6753 \\
\hline Total & 12 & & & & & & & \\
\hline
\end{tabular}

En la prueba de solubilidad del extracto atomizado y liofilizado de la Ipomea batata L., camote morado. Los resultados fueron: en agua y etanol son miscibles, en metanol no son miscibles, según se demuestra en la tabla 1. Observando resultados similares en Saucedo JC. (2016), quien investigó en las pruebas de ensayo de solubilidad en "Obtención de antioxidantes en polvo a partir de la Ipomea batata L. (camote morado)". Se demostró que la mayor solubilidad en solventes polares fue agua y etanol. Lo que nos indicaría la presencia mayoritaria de compuestos fenólicos de alta polaridad por lo que se puede inferir que los componentes químicos son de estructura y naturaleza polar, los cuales fueron corroborados previamente por Saucedo JC. (2016).

Según los resultados de la actividad antioxidante por el método DPPH se obtuvo $23,23 \mu \mathrm{M}$ Trolox/g en el camote morado liofilizado y 5,24 $\mu \mathrm{M}$ Trolox/g en el obtenido por atomizado, en comparación con el autor Cervantes R. (2019), que obtuvo por el método de DPPH valores de 25,16 $\mu \mathrm{mol}$ Trolox/g en hojas de camote morado. De acuerdo a estos resultados el Ipomoea batata L., camote morado, Cervantes R. (2019) tuvo resultados con mayor contenido de capacidad antioxidante en comparación al presente trabajo.

Al respecto de los resultados obtenidos en el presente trabajo respecto a la actividad antioxidante por el método FRAP, se presentan los resultados de 4,96 y 20,18 ( $\mu \mathrm{M}$ Trolox/g) en el camote morado atomizado y liofilizado, respectivamente y según el autor Cervantes R. (2019) utilizando el método de capacidad antioxidante equivalente a Trolox con el ácido 2,2'-azino-bis-3-etilbenzotiazolina-6-sulfónico, obtuvo 8,61 $\mu \mathrm{mol}$ Trolox/mL. Según estos resultados el Ipomoea batata L., camote morado presenta mayor contenido de capacidad antioxidantes en el presente estudio frente al autor Cervantes R. (2019). 
En referencia a los resultados de capacidad antioxidante obtenido por el método de DPPH $5,2407 \mu \mathrm{M}$ Trolox/g y 4,9575 $\mu \mathrm{M}$ Trolox/g por el método FRAP en la muestra deshidratada por atomización, no se puede observar una diferencia considerable aun teniendo diferentes técnicas de análisis. Esto se corresponde con los resultados obtenidos en las muestras deshidratadas por liofilización: 23,2251 $\mu \mathrm{M}$ Trolox/g analizada por el método de DPPH y 20,1814 $\mu \mathrm{M}$ Trolox/g por el método de FRAP que también reflejan resultados semejantes.

Los resultados de capacidad antioxidante por ambos métodos DPPH y FRAP, utilizados en el presente trabajo, muestran una gran variación debido al tipo de deshidratación al cual se sometió la muestra de Ipomoea batata L., camote morado. Esto demuestra que el proceso para estabilizar la muestra afecta de manera considerable y es que la elevada temperatura podría afectar sus componentes, lo que no ocurre con el proceso de liofilizado que utiliza la deshidrocongelación, de esta forma congelando y reduciendo la presión, reflejando la diferencia en los mejores resultados de la capacidad antioxidante

\section{CONCLUSIONES}

Se puede concluir que la muestra de camote morado liofilizada presentó mayor contenido de capacidad antioxidante en cuanto a la muestra atomizada que presenta valores más bajos, por lo cual es importante resaltar que el proceso de deshidratación afecta en gran medida los resultados obtenidos.

Comparando los métodos de DPPH y FRAP para los diferentes procesos de deshidratación de la muestra de Ipomoea Batata L., camote morado, se pudo observar que los resultados se corresponden reflejando que ambos métodos de análisis tienen resultados semejantes utilizando muestras deshidratadas por el mismo proceso.

\section{REFERENCIAS BIBLIOGRÁFICAS}

1. Llancari A, Matos A. Valoración de los nutrientes y antioxidantes en la salud humana e industria alimentaria. Paper presentado en: I Congreso Nacional de Investigación, Universidad Peruana Unión, noviembre 2011; Lima, Perú.

2. Núñez A. Terapia antioxidante, estrés oxidatívo y productos antioxidantes: retos y oportunidades. Rev Cub Salud Pública. 2011; 37: 644-60.

3. Ghasemzadeh A, Omidvar V, Hawa Z. Polyphenolic content and their antioxidant activity in leaf extract of sweet potato (Ipomoea batata). J Med Plants Res. 2012; 6(15): 2971-2976.

4. Seow-Mun H, Amru N, Chandran S. Antioxidant activity, phenolic and flavonoid contents in the leaves of different varieties of sweet potato (Ipomoea batata). Aust $\mathbf{J}$ Crop Sci. 2012; 6(3): 375-380.

5. Vizzotto M, Dos Santos E, Rocha J, Cardoso P, Lettnin M, Suita L, Richter A. Physicochemical and antioxidant capacity analysis of colored sweet potato genotypes: 
in natura and thermally processed. Ncia Rural. 2017; 47: 04. doi: 10.1590/01038478 cr20151385

6. Del Carpio BR. Selecciones y variedades de camote. La vida agrícola. Asesor del CRIA I la Molina, Lima. 1980.

7. Ruttarattanamongkol K, Chittrakorn S, Weerawatanakorn M, Dangpium N. Effect of drying conditions on properties, pigments and antioxidant activity retentions of pretreated orange and purple-fleshed sweet potato flours. J Food Sci Technol. 2016; 53(4): 1811-1822.

8. Bellail A, Shaltout O, Youssef M, Gamal A. Effect of Home-Cooking Methods on Phenolic Composition and Antioxidant Activity of Sweetpotato (Ipomoea batata (L.) Lam.) Cultivars Grown in Egypt. Food Nutr Sci. 2012; 3: 490-499.

9. Yang J, Chen J, Zhao Y, Mao L. Effects of Drying Processes on the Antioxidant Properties in Sweet Potatoes. Agr Sci China. 2010; 9(10): 1522-1529.

10. Avello M. Suwalsky M. Radicales libres, antioxidantes naturales y mecanismos de protección. Atenea (Concepción). 2006; 494: 161-172.

11. Vidal A, Zaucedo A, Ramos M. Propiedades nutrimentales del camote (Ipomoea batata L.) y sus beneficios en la salud humana. Revista Iberoamericana de Tecnología Postcosecha. [Internet]. 2018 [Citado 15 nov 2020]: 19(2). Disponible en: https://www. redalyc.org/jatsRepo/813/81357541001/81357541001.pdf

12. Saucedo J. Obtencion de Antioxidantes en polvo a partir de la (Ipomoea batata, camote morado). [Tesis Ingeniero Químico]. Trujillo: Universidad Nacional de Trujillo, E.A.P. Ingeniería Química; 2016.

13. Cervantes R, Barragán M, Chaquilla G. Evaluación de antioxidantes en el té de hojas de camote morado (Ipomoea batatas L.). Tecnología en Marcha. 2019; 32: 51-59. 